\title{
Evaluation and management of acute abdominal pain in the emergency department
}

This article was published in the following Dove Press journal:

International Journal of General Medicine

24 September 2012

Number of times this article has been viewed

\section{Christopher R Macaluso \\ Robert M McNamara \\ Department of Emergency Medicine, Temple University School of Medicine, Philadelphia, PA, USA}

\begin{abstract}
Evaluation of the emergency department patient with acute abdominal pain is sometimes difficult. Various factors can obscure the presentation, delaying or preventing the correct diagnosis, with subsequent adverse patient outcomes. Clinicians must consider multiple diagnoses, especially those life-threatening conditions that require timely intervention to limit morbidity and mortality. This article will review general information on abdominal pain and discuss the clinical approach by review of the history and the physical examination. Additionally, this article will discuss the approach to unstable patients with abdominal pain.
\end{abstract}

Keywords: acute abdomen, emergency medicine, peritonitis

\section{Introduction}

Abdominal pain is the most common reason for a visit to the emergency department (ED), accounting for 8 million (7\%) of the 119 million ED visits in 2006. ${ }^{1}$ Obviously, anyone practicing emergency medicine (EM) must be skilled in the assessment of abdominal pain. Although a common presentation, abdominal pain must be approached in a serious manner, as it is often a symptom of serious disease and misdiagnosis may occur. Abdominal pain is the presenting issue in a high percentage of medicolegal actions against both general and pediatric EM physicians. ${ }^{2,3}$ The modern physician should be humbled by the fact that, despite diagnostic and therapeutic advances (computed tomography [CT], ultrasonography, and laparoscopy), the misdiagnosis rate of the most common surgical emergency, acute appendicitis, has changed little over time. ${ }^{4}$

\section{History}

The clinician should try to obtain as complete a history as possible as this is generally the cornerstone of an accurate diagnosis. The history should include a complete description of the patient's pain and associated symptoms. Medical, surgical, and social history should also be sought as this may provide important information.

\section{Assessment of the patient's pain}

The classic PQRST mnemonic for a complete pain history is as follows:

P3 - Positional, palliating, and provoking factors

Q - Quality

R3 - Region, radiation, referral

$\mathrm{S}-$ Severity

T3 - Temporal factors (time and mode of onset, progression, previous episodes) 
This mnemonic will help to ensure a thorough history, but rigidly following the above sequence does not allow for a smooth patient interview, so the authors prefer to ask the patient where they feel the pain (location), what kind of pain it is (character), when and how it began (onset), how bad it is (intensity), and where else they feel it, what makes it worse or better, how it has changed over time, and whether they have ever had it before.

\section{Location}

Embryology determines where a patient will "feel" visceral pain, which is generally perceived in the midline because afferent impulses from visceral organs are poorly localized. Visceral nociceptors can be stimulated by distention, stretch, vigorous contraction, and ischemia. Pain from foregut structures, which include the stomach, pancreas, liver, biliary system, and the proximal duodenum, will be typically localized to the epigastric region. The rest of the small bowel and the proximal third of the colon including the appendix are midgut structures, and visceral pain associated with these organs is perceived in the periumbilical region. Hindgut structures such as the bladder, and distal two-thirds of the colon, as well as pelvic genitourinary organs usually cause pain in the suprapubic region. Pain is usually reported in the back for retroperitoneal structures such as the aorta and kidneys. ${ }^{5,6}$

\section{Character}

Clinicians should seek to distinguish between the dull, poorly localized, aching, or gnawing pain generated by viscerally innervated organs, compared with the characteristically "sharp", more defined and localized somatic pain caused by irritation of the parietal peritoneum or other somatically innervated structures. Somatic pain is transmitted via the spinal nerves from the parietal peritoneum or mesodermal structures of the abdominal wall. Noxious stimuli to the parietal peritoneum may be inflammatory or chemical in nature (eg, blood, infected peritoneal fluid, and gastric contents). ${ }^{5,7}$

\section{Onset}

Acute-onset pain, especially if severe, should prompt immediate concern about a potential intra-abdominal catastrophe. The foremost consideration would be a vascular emergency such as a ruptured abdominal aortic aneurysm (AAA) or aortic dissection. Other considerations for pain of acute onset include a perforated ulcer, volvulus, mesenteric ischemia, and torsion; however, these conditions may also occur without an acute onset. For example, only $47 \%$ of elderly patients with a proven perforated ulcer report the acute onset of pain. ${ }^{8}$ Likewise, volvulus, particularly of the sigmoid colon, can present with a gradual onset of pain. ${ }^{9}$ Serious vascular issues such as mesenteric ischemia may present with a gradual onset of pain. Conversely, one would expect a gradual onset in the setting of an infectious or inflammatory process. Pain that awakens the patient from sleep should be considered serious until proven otherwise. ${ }^{10}$ The time of onset establishes the duration of the pain and allows the physician to interpret the current findings in relation to the expected temporal progression of the various causes of abdominal pain.

\section{Intensity}

Pain that is severe should heighten the concern for a serious underlying cause; however, descriptions of milder pain cannot be relied on to exclude serious illness, especially in older patients who may under-report symptoms.

\section{Patterns of radiation and referral of pain}

The neural pathways give rise to predictable patterns of referred pain and radiation. Kehr's sign is a classic example where diaphragmatic irritation, usually from free intraperitoneal blood, causes shoulder pain. ${ }^{11}$ Any other inflammatory process or organ contiguous to the diaphragm can also cause referred shoulder pain. Another well described example is ipsilateral scapula pain caused by biliary disease. Radiation may also reflect progression of disease such as with continued aortic dissection, or ongoing passage of a ureteral stone. While considering referred pain, it is important to remember that deep musculoskeletal structures (especially of the back) are innervated by visceral sensory fibers with similar qualities to those arising from intra-abdominal organs. These fibers converge in the spinal cord, giving rise to "scleratomes": regions of referral in the abdomen and flanks. Thus, in cases where a patient's perceived location of symptoms appears to be completely unrevealing on physical exam, a careful assessment of musculoskeletal structures should be made. ${ }^{12}$

\section{Duration and progression}

Persistent worsening pain is worrisome, while pain that is improving is typically favorable. Serious causes of abdominal pain generally present early in their course; however, delays in presentation can occur, especially in the elderly. Certain patterns of progression can be diagnostic, such as the migration of pain in appendicitis where the initial distention of the appendix causes a periumbilical visceral pain that shifts to the right lower quadrant once the inflammatory process is 
detected by the somatic sensors of the parietal peritoneum. Although labeled "colic," gallbladder pain is generally not paroxysmal, and it almost never lasts less than 1 hour, with an average of 5-16 hours' duration, and ranging up to 24 hours. ${ }^{13}$ Small bowel obstruction typically progresses from an intermittent ("colicky") pain to more constant pain when distention occurs. One would only expect somatic pain (arising from transmural ischemia or perforation contiguous to the parietal peritoneum) late in the course of a bowel obstruction.

\section{Provocative and palliating factors}

The clinician needs to ask what, if anything, makes the pain worse and what improves the pain. It is important to establish whether jarring motions such as coughing or walking exacerbate the pain, suggesting peritoneal irritation. ${ }^{14}$ Patients with peritonitis often remark on increased pain with jolts or bumps in the road. A recommended interview sequence would be "how did you get here today? Tell me about the ambulance/car ride." With upper abdominal pain one should specifically ask whether it is pleuritic as this may signify chest disease. Peptic ulcer disease may be exacerbated (gastric) or relieved (duodenal) by eating. Mesenteric ischemia may be precipitated by eating, as can the pain of intermittently symptomatic gallstones, often associated with fatty meals. The patient should be questioned about any self treatments, particularly analgesics and antacids, and the response to these measures.

\section{Previous episodes}

Recurrent episodes generally point to a medical cause, with the exceptions of mesenteric ischemia (intestinal angina), gallstones, or partial bowel obstruction.

\section{Assessment of the associated symptoms}

Gastrointestinal and urinary symptoms are the primary focus; however, it is important to ask about fever and cardiopulmonary symptoms. Associated symptoms should be placed in the clinical context, including the patient's age and the current point in the course of the illness.

\section{Anorexia}

With appendicitis, most physicians expect the patient to report anorexia. However, pooling of the literature indicates that, while this is a discriminatory symptom, it is only present in $68 \%$ of patients with appendicitis. ${ }^{15}$ The report of this symptom decreases to $20 \%-44 \%$ in elderly patients with appendicitis. $^{16}$

\section{Vomiting}

Vomiting may occur in almost any abdominal disease. Pain generally precedes vomiting in surgical conditions, with the important exception of esophageal rupture from forceful emesis. ${ }^{10,17}$ It is usually present in small bowel obstruction, unless the obstruction is partial or the patient is presenting early in the course. Many other serious entities including large bowel obstruction frequently present without vomiting. The nature of the vomiting may be diagnostically helpful. With small bowel obstruction, one anticipates a progression from gastric contents to bilious to feculent emesis as the duration of the illness increases. Frequent nonproductive retching can point to gastric volvulus, ${ }^{18}$ while repetitive nonbilious vomiting may indicate gastric outlet obstruction. The presence of blood or bile should be noted. Bilious vomiting in an infant is always considered a harbinger of serious abdominal illness such as intestinal malrotation. ${ }^{19}$ Blood or coffee ground emeses are usually caused by gastric diseases or complications of liver disease. Massive hematemesis in a patient with a prior abdominal aortic aneurysm repair may be due to aorto-enteric fistula. ${ }^{20}$ The key feature of vomiting from more benign causes such as viral or food-borne illness is that it is self-limited.

\section{Bowel symptoms}

While diarrhea is a frequent accompaniment of more benign abdominal conditions, its presence alone should never rule out serious disease. For example, diarrhea is common with mesenteric ischemia and is frequently reported in conditions such as appendicitis. ${ }^{15,21}$ In one series of 1000 ED patients presenting with abdominal pain, $18 \%$ presented with diarrhea. No patient aged less than 40 with diarrhea and continuous pain was found to have a surgical cause for their symptoms. ${ }^{22}$ Conversely, diarrhea can be reported in up to one-fifth of patients with colonic obstruction. ${ }^{23}$ Diarrhea also occurs in early small bowel obstruction as the reflexively hyperactive bowel distal to the obstruction clears itself, and with partial obstruction, diarrhea may be ongoing. Absence of flatus is a more reliable sign than constipation in bowel obstruction, since the bowel clears gas more rapidly than fluid. Bloody stool in the presence of significant abdominal pain should raise the suspicion for mucosal compromise from ischemia. Melena suggests an upper source of bleeding, while frank blood can indicate a lower source or a massive upper bleed with rapid transit time. The urge to defecate in a patient with acute abdominal pain has been described as a harbinger of serious disease including a ruptured aneurysm in the older patient or ruptured ectopic pregnancy in the young. ${ }^{24}$ 


\section{Other symptoms}

Many genitourinary tract diseases can present with abdominal pain. Conversely, any inflammatory process contiguous to the genitourinary tract (including appendicitis, cholecystitis, pancreatitis, or any inflammatory process involving bowel) may result in both pyuria and dysuria. This can lead to misdiagnosis of both gastrointestinal and genitourinary conditions. In men, testicular torsion may present as abdominal pain, nausea, and vomiting. In women, the enlarging uterus of pregnancy can itself cause discomfort and displace abdominal organs in such a way as to further complicate the diagnosis of many abdominal conditions, especially appendicitis. For these reasons, a menstrual (where applicable), sexual, and genitourinary history should be obtained in most patients with abdominal pain. The report of normal, regular menses should not preclude consideration of current pregnancy. ${ }^{25}$ Cardiopulmonary symptoms such as cough and dyspnea can point to a nonabdominal cause of abdominal pain. Syncope may indicate disease originating in the chest (pulmonary embolism, dissection) or abdomen (acute aortic aneurysm, ectopic pregnancy).

\section{Past medical and surgical history, current medications}

A history of prior abdominal surgery can rule out a condition or raise the suspicion for a complication such as obstruction from adhesions. Many medical conditions cause acute abdominal pain, including diabetic ketoacidosis, hypercalcemia, Addison's disease, and sickle cell crisis. Other less common metabolic causes of acute abdominal pain include uremia, lead poisoning, methanol intoxication, hereditary angioedema, and porphyria. The patient's current medications should be reviewed with attention to those affecting the integrity of the gastric mucosa (steroids and nonsteroidal anti-inflammatory drugs), immunosuppressive agents (impair host defenses and the generation of an inflammatory response), and any that can impair nociception (narcotics may also be a cause of pain due to constipation).

\section{Social history}

The patient's use of drugs and alcohol may have important diagnostic implications. Cocaine use may cause intestinal as well as cardiac ischemia. Gastrointestinal complications of alcohol abuse are extensive and well known. Direct questions regarding domestic violence may reveal a traumatic source of pain.

\section{Physical examination}

The general appearance of the patient is noted first. An "ill appearing" patient with abdominal pain is always of great concern given the variety of potentially lethal underlying causes. On the other hand, especially in the elderly, the clinician must not be misled by the well appearing patient who may still have serious underlying disease. ${ }^{26}$ The clinician should take note of the patient's position, spontaneous movements, respiratory pattern, and facial expression.

\section{Vital signs}

Vital sign abnormalities should alert the clinician to a serious cause of the abdominal pain. However, the presence of normal vital signs does not exclude a serious diagnosis. While fever certainly points to an infectious cause or complication, it is frequently absent with infectious causes of abdominal pain. For example, fever is absent in over $30 \%$ of patients with appendicitis and in the majority of those with acute cholecystitis. ${ }^{15,27}$ Tachypnea may be a nonspecific finding, but it should prompt consideration of chest disease or metabolic acidosis from entities such as ischemic bowel or diabetic ketoacidosis.

\section{The abdominal examination}

The emergency physician should know the key elements of the abdominal examination while understanding their limitations. In particular, all techniques for the detection of peritonitis will yield both false-negative and false-positive results.

\section{Inspection, auscultation, and percussion}

Inspection is important for the detection of surgical scars, skin changes including signs of herpes zoster, liver disease (caput medusa), and hemorrhage (Grey Turner's sign of flank ecchymoses with a retroperitoneal source, Cullen's sign of a bluish umbilicus with intraperitoneal bleeding). ${ }^{11}$ With distention, percussion will allow the differentiation between large bowel obstruction (drum-like tympany) and advanced ascites (shifting dullness). Auscultation is of very limited diagnostic utility, and prolonged listening for bowel sounds is an ineffective use of time, although it may reveal high pitched sounds in early small bowel obstruction or the silence encountered with ileus or late in the course of any abdominal catastrophe. Bruits have been described with aortic, renal, or mesenteric stenosis, but are rarely appreciated in a busy ED. ${ }^{28}$

\section{Palpation}

The ED abdominal exam is directed primarily to the localization of tenderness, the identification of peritonitis, and the detection of certain enlargements such as the 
abdominal aorta. Various strategies have been advocated to improve the palpation phase of the examination, including progression from nonpainful areas to the location of pain. It may be useful to palpate the abdomen of anxious or less cooperative children with the stethoscope to define areas of tenderness. ${ }^{29}$ Meyerowitz ${ }^{30}$ advocates following up the initial examination with a secondary palpation with a stethoscope while telling the patient one is listening in order to uncover exaggerated symptoms. It is preferable to have the patient flex the knees and hips to allow for relaxation of the abdominal musculature (see below discussion of guarding).

Localized tenderness is generally a reliable guide to the underlying cause of the pain. More generalized tenderness presents a greater diagnostic challenge. Unless the patient has had an appendectomy, the authors recommend, given its frequency as a serious cause of abdominal pain, continued consideration of appendicitis in any patient with right lower quadrant tenderness. Despite the known issues with diagnosing appendicitis in the elderly, virtually all of them will have right lower quadrant tenderness. ${ }^{8}$ If tolerated by the patient, palpation or percussion may include assessment of the liver and spleen size and a search for pulsatile or other masses and an assessment of the quality of femoral pulses. A tender pulsatile and expansile mass is the key distinguishing feature of an acute abdominal aortic aneurysm, although this and most other masses are much more accurately diagnosed with the aid of a bedside ultrasound machine, if available. ${ }^{31}$ The femoral pulses may be unequal with aortic dissection. ${ }^{32}$ Inspection and palpation of the patient while they are standing may reveal the presence of hernias undetected in the supine position.

\section{Tests for peritoneal irritation}

Determining the presence or absence of peritonitis is a primary objective of the abdominal examination; unfortunately, the methods for detecting it are often inaccurate. Traditional rebound testing is performed by gentle depression of the abdominal wall for approximately $15-30$ seconds with sudden release. The patient is asked whether the pain was greater with downward pressure or with release. Despite limitations, the test was one of the most useful in a meta-analysis of articles investigating the diagnosis of appendicitis in children. ${ }^{29}$ Cope's Early Diagnosis of the Acute Abdomen recommends against this test because it is unnecessarily painful. The authors suggest gentle percussion as more accurate and humane. ${ }^{28}$ When subject to study, traditional rebound testing has a sensitivity for the presence of peritonitis near $80 \%$, yet its specificity is only $40 \%-50 \%$ and it is entirely nondiscriminatory in the identification of cholecystitis. ${ }^{27,33,34}$ The use of indirect tests such as the "cough test," where one looks for signs of pain such as flinching, grimacing, or moving the hands to the abdomen upon coughing has a similar sensitivity but with a specificity of $79 \% .{ }^{35}$ In children, indirect tests would include the "heel drop jarring" test (child rises on toes and drops weight on heels) or asking the child to jump up and down while looking for signs of abdominal pain. ${ }^{29,36}$

Guarding is defined as increased abdominal wall muscular tone and is only of significance as an involuntary reflex when it reflects a physiological attempt to minimize movement of the intraperitoneal structures. In contrast, "voluntary guarding" can be induced by any person with conscious control of their abdominal wall musculature and is frequently seen in completely normal patients with apprehension about the abdominal exam. Rigidity is the extreme example of true guarding. To identify true guarding, the examiner gently assesses muscle tone through the respiratory cycle, preferably with the knees and hips flexed to further relax the abdomen. With "voluntary guarding," the tone will decrease with inspiration, while with true guarding, the examiner will be able to detect continued abdominal wall tension throughout the respiratory cycle. Guarding and rigidity may be lacking in the elderly because of laxity of the abdominal wall musculature. Disturbingly, only $21 \%$ of patients over the age of 70 with a perforated ulcer presented with epigastric rigidity. ${ }^{8}$

\section{The rectal examination}

The diagnostic value of a rectal examination in the evaluation of acute abdominal pain is limited; however, it may be of use in detecting intestinal ischemia, late intussusception, or colon cancer. The routine performance of a rectal examination in suspected appendicitis is not supported by the available literature. ${ }^{37}$ It is recommended that, as a general rule, one should not perform this examination in children as it adds little to the diagnostic process at the cost of significant discomfort. ${ }^{38}$ On the other hand, the exam's utility is likely to increase with the patient's age, and one study found that within 1 year nearly $11 \%$ of patients over the age of 50 diagnosed with nonspecific abdominal pain in an ED were found to have cancer, principally of the colon. ${ }^{33}$ The use of the rectal examination in other age groups should be targeted to diagnoses in which it may yield important information. ${ }^{37}$

\section{Special abdominal examination techniques}

There are a number of examination techniques that may be useful to the emergency physician in helping to establish a diagnosis. 
Some of these tests have not been well studied, but documentation of their presence or absence on the chart will indicate a consideration of a specific disease process such as appendicitis.

\section{Carnett's sign}

Abdominal wall tenderness can be caused by trauma, and with increasing numbers of patients on therapeutic anticoagulation, abdominal wall hematoma. The following technique, described by Carnett in 1926, may confirm the abdominal wall as the source of the patient's pain. The point of maximal pain is identified, and this is palpated with the abdomen wall relaxed and then tensed through the performance of a half sit-up with the arms crossed. Increased pain with the wall tensed is a positive sign of abdominal wall pathology, a decrease in pain is considered a negative test. When prospectively applied in 120 patients, the test was positive in 24 , with only one having an intra-abdominal pathologic condition. ${ }^{39}$ Others have found it less accurate but still useful. ${ }^{40}$ This test should not be routinely applied but is considered when there is a supportive history and absence of indicators of other illness. ${ }^{41}$

\section{Cough test}

Originally described by Rostovzev in 1909, this test seeks evidence of peritoneal irritation by having the patient cough. ${ }^{42}$ Jeddy and colleagues ${ }^{43}$ described a positive test as a cough causing a sharp, localized pain. They applied this prospectively to patients with right lower quadrant pain and found it to have near perfect sensitivity with a specificity of $95 \%$ for the detection of appendicitis or peritonitis (one patient with perforated diverticulitis). Bennett and colleagues ${ }^{35}$ consider signs of pain on coughing such as flinching, grimacing, or moving of hands to the abdomen as a positive test and reported a sensitivity of $78 \%$ with a specificity of $79 \%$ for the detection of peritonitis in a prospective study of 150 consecutive patients with abdominal pain.

\section{Closed eyes sign}

Based on the assumption that the patient with an acute abdominal condition will carefully watch the examiner's hands to avoid unnecessary pain, this test is considered an indicator of nonorganic cause of abdominal pain. The test is considered positive if the patient keeps their eyes closed when abdominal tenderness is elicited. In a prospective study of 158 patients, Gray and colleagues ${ }^{44}$ found that $79 \%$ of the 28 patients who closed their eyes did not have identifiable organic pathology.

\section{Murphy's sign}

Murphy described cessation of inspiration in cholecystitis when the examiner curled their fingers below the anterior right costal margin from above the patient. ${ }^{45}$ Now most commonly performed from the patient's side, inspiratory arrest while deeply palpating the right upper quadrant is the most reliable clinical indicator of cholecystitis, although it only has a sensitivity of $65 \% .^{27}$

\section{The psoas sign}

The psoas sign is provoked by having the supine patient lift the thigh against hand resistance, or with the patient laying on their contralateral side and the hip joint passively extended. Increased pain suggests irritation of the psoas muscle by an inflammatory process contiguous to the muscle. When positive on the right, this is a classic sign suggestive of appendicitis. Other inflammatory conditions involving the retroperitoneum, including pyelonephritis, pancreatitis, and psoas abscess, will also elicit this sign.

\section{The obturator sign}

The obturator sign is elicited with the patient supine and the examiner supporting the patient's lower extremity with the hip and knee both flexed to 90 degrees. The sign is positive if passive internal and external rotation of the hip causes reproduction of pain, and suggests the presence of an inflammatory process adjacent to the muscle deep in lateral walls of the pelvis. Potential diagnoses include a pelvic appendicitis (on the right only), sigmoid diverticulitis, pelvic inflammatory disease, or ectopic pregnancy.

\section{The Rovsing sign}

The Rovsing sign is a classic test used in the diagnosis of appendicitis. It is a form of indirect rebound testing in which the examiner applies pressure in the left lower quadrant, remote from the usual area of appendiceal pain and tenderness. The test is positive if the patient reports rebound pain in the right lower quadrant when the examiner releases pressure. ${ }^{15}$ In limited studies, the psoas, obturator, and Rovsing signs demonstrate a low sensitivity $(15 \%-35 \%)$ but a relatively high specificity (85\%-95\%) for appendicitis..$^{15,29,46}$

\section{Other examination elements}

Careful examination of adjacent areas is a key part of the assessment of the patient with abdominal pain. In addition to skin inspection, the back should be assessed for tenderness at the costovertebral angle, spinous processes, and paraspinal regions. Because virtually any chest disease can present 
with abdominal pain, particular attention should be paid to the cardiopulmonary examination. The groin, including the femoral triangle, is assessed for hernias. The male patient must be inspected for testicular pathology including torsion and infection. In females with lower abdominal pain a pelvic examination is almost always necessary. The pelvic examination presents an opportunity to assess the pelvic peritoneum directly for signs of inflammation through the assessment of cervical motion tenderness. If Fitzhugh-Curtis syndrome is a consideration, a pelvic examination may be indicated with upper abdominal pain. ${ }^{7}$

\section{Analgesia and the abdominal examination}

The emergency physician should not hesitate to administer adequate analgesic medication to the patient with acute abdominal pain. When studied, the administration of narcotic analgesics does not obscure the diagnosis or interfere with the treatment of the patient. The United States Agency for Healthcare Research and Quality issues reports regarding making health care safer and recommended this practice after a review of the literature. ${ }^{47}$ Previously, Cope's Early Diagnosis of the Acute Abdomen admonished the physician for administering morphine, but this stance has been reversed in more recent editions. ${ }^{47,48}$ Thomas and colleagues ${ }^{49}$ authored a prospective study where the administration of up to $15 \mathrm{mg}$ of morphine did not affect diagnostic accuracy in patients with acute abdominal pain. They further recommended its use in that it fulfills the physician's "imperative to relieve suffering."

\section{Approach to the unstable patient}

On occasion, a patient with acute abdominal pain will present in extremis. The ill-appearing patient with abdominal pain requires immediate attention. This is particularly so in the elderly, as the overall mortality rate for all older patients with acute abdominal pain ranges from $11 \%-14 \%$, and those presenting in an unstable fashion have an even poorer prognosis. $^{26}$

The usual sequence of resuscitation is applied to the unstable abdominal pain patient with airway control achieved as necessary. Hypotension requires the parallel process of treatment and an early assessment for life threatening conditions requiring emergent surgical intervention. Hypotension from blood and fluid loss from the gastrointestinal tract is usually apparent from the history coupled with a digital rectal examination. If this evidence is lacking in the patient with abdominal pain, there needs to be early consideration of third spacing, which can cause enormous fluid shifts into the bowel lumen or peritoneal space in bowel obstruction or other intestinal catastrophes. Bedside ultrasonography is an extremely useful diagnostic adjunct in such patients. In the older patient, hypotension should prompt an immediate search for an abdominal aortic aneurysm, immediately followed by sonographic evaluation of the inferior vena cava for intravascular volume status, and sonography of the heart, pleural, and peritoneal spaces to exclude massive effusions or evidence of massive pulmonary embolus. Bedside echocardiography will also identify severe global myocardial depression as a cardiogenic cause of shock. In the younger patient, a large amount of free fluid detected by ultrasound in an unstable patient is most commonly due to rupture of an ectopic pregnancy, spleen, or hemorrhagic ovarian cyst. An immediate urine pregnancy test will be the first step in distinguishing these.

The proper place for the unstable patient with an acute abdominal aortic aneurysm is the operating room or, in some centers, the interventional suite for emergency aortic stent placement. Attempts to obtain CT imaging, may cause fatal delays in definitive treatment. With a high clinical index of suspicion (if possible, supported by emergency bedside ultrasonography), most patients sent directly to surgery will be found to have an acute AAA, and nearly all others will have an alternative diagnosis that still needs operative intervention. ${ }^{50}$

\section{Diagnostic studies and disposition}

Appropriate diagnostic testing is covered in the respective chapters for specific entities; however, it must be emphasized that there are significant limitations of imaging and laboratory studies in the evaluation of acute abdominal pain and all diagnostic tests have a false-negative rate. If the history and physical examination leads to a high pre-test probability of a disease, a negative test cannot exclude the diagnosis. For example, the total leukocyte count can be normal in the face of serious infection such as appendicitis or cholecystitis. ${ }^{29,51}$ CT is frequently used in evaluation of the patient with abdominal pain. Clinicians are enamored with the recent advances in the technology that have allowed for improved image resolution and shorter acquisition times along with coronal and three-dimensional reconstruction. However, it remains an imperfect test for conditions such as appendicitis and may add little to the clinical assessment. ${ }^{52,53}$

Plain abdominal radiographs are of limited utility in the evaluation of acute abdominal pain. ${ }^{54}$ Although they may be helpful (free intraperitoneal air, calcified aortic aneurysm, air fluid levels in obstruction) other diagnostic 
studies are almost always indicated or perform better as the initial testing. If plain radiographs are utilized, the limitations must be appreciated. For example, a standard upright film will not demonstrate free air in up to $40 \%$ of patients with a perforated ulcer. ${ }^{55}$

The oft repeated axiom of "treat the patient, not the test" certainly applies in the patient with acute abdominal pain. An unexpected negative test result should prompt a reassessment of the patient and consideration for observation and repeat examination for disease progression. Whenever the diagnosis is in question, serial examination as an inpatient in an observation unit or in the ED is a sound strategy. When a patient is discharged home after an evaluation for abdominal pain, the authors recommend instructions to return if the pain worsens, new vomiting or fever occurs, or if the pain persists beyond 8-12 hours. Such instructions are targeted at ensuring the return of a patient who has progressed from an early appendicitis or small bowel obstruction, the two most common surgical entities erroneously discharged from an ED. ${ }^{11,17}$

\section{Disclosure}

The authors report no conflicts of interest in this work.

\section{References}

1. Pitts SR, Niska RW, Xu J, et al. National hospital ambulatory medical care survey: 2006 emergency department summary. National health statistics report; no 7. Hyattsville, MD: National Center for Health Statistics; 2008.

2. Selbst SM, Friedman MJ, Singh SB. Epidemiology and etiology of malpractice lawsuits involving children in US emergency departments and urgent care centers. Pediatr Emerg Care. 2005;21:165-169.

3. Kachalia A, Gandhi TK, Puopolo AL, et al. Missed and delayed diagnoses in the emergency department: a study of closed malpractice claims from 4 liability insurers. Acad Emerg Med. 2007;49:196-205.

4. Flum DR, Morris A, Koepsell T, et al. Has misdiagnosis of appendicitis decreased over time? JAMA. 2001;286:1748-1753.

5. Jung PJ, Merrell RC. Acute abdomen. Gastroenterol Clin North Am. 1988; 17:227-244.

6. Jones RS, Claridge JA. Acute abdomen. In: Townsend CM, Beauchamp RD, Evers BM, et al, editors. Sabiston Textbook of Surgery: the Biologic basis of Modern Surgical Practice. 17th ed. Philadelphia: Elsevier; 2004:1219-1238.

7. Abbott J. Pelvic pain: lessons from anatomy and physiology. J Emerg Med. 1990;8:441-447.

8. Fenyo G. Acute abdominal disease in the elderly: experience from two series in Stockholm. Am J Surg. 1982;143:751-754.

9. Anderson JR, Lee D. The management of acute sigmoid volvulus. Br J Surg. 1981;68:117-120.

10. Silen W. Method of diagnosis: the history. In: Cope's Early Diagnosis of the Acute Abdomen. New York: Oxford; 2010:18-27.

11. Hickey MS, Kiernan GJ, Weaver KE. Evaluation of abdominal pain. Emerg Med Clin North Am. 1989;7:437-452.

12. Feinstein B, Langton JNK, Jameson RM, et al. Experiments on pain referred from deep somatic tissues. J Bone Joint Surg Am. 1954;36: 981-997.
13. Silen W. Cholecystitis and other causes of acute pain in the right upper quadrant of the abdomen. In: Cope's Early Diagnosis of the Acute Abdomen. New York: Oxford; 2010:131-140.

14. Giamberardino MA, De Laurentis S, Affaitati G, et al. Modulation of pain and hyperalgesia from the urinary tract by algogenic conditions of the reproductive organs in women. Neurosci Lett. 2001;304(1-2): 61-64.

15. Wagner JM, McKinney WP, Carpenter JL. Does this patient have appendicitis? JAMA. 1996;2786:1589-1594.

16. Kraemer M, Franke C, Ohmann C, et al. Acute appendicitis in late adulthood: incidence, presentation, and outcome. Results of a prospective multicenter acute abdominal pain study and a review for the literature. Arch Surg. 2000;3835:470-481.

17. Brewer RJ, Golden GT, Hitch DC, et al. Abdominal pain: an analysis of 1,000 consecutive cases in a university hospital emergency room. Am J Surg. 1976;131:219-224.

18. Godshall D, Mossallam W, Rosenbaum R. Gastric volvulus: case report and review of the literature. J Emerg Med. 1999;17:837-840.

19. Schafermeyer RW. Pediatric abdominal emergencies. In: Tintinalli JE, Kelen GD, Stapczynski S, et al, editors. Emergency Medicine: a Comprehensive Study Guide. 6th ed. New York: McGraw-Hill; 2004:844-851.

20. Busuttil SJ, Goldstone J. Diagnosis and management of aortoenteric fistulas. Semin Vasc Surg. 2001;14:302-311.

21. Inderbitzi R, Wagner HE, Seiler C, et al. Acute mesenteric ischaemia. Eur J Surg. 1992;158:123-126.

22. Chen EH, Shofer FS, Dean AJ, et al. Derivation of a clinical prediction rule for evaluating patients with abdominal pain and diarrhea. $\mathrm{Am} \mathrm{J}$ Emerg Med. 2008;26(4):450-453.

23. Greenlee HB, Pienkos EJ, Vamderbilt PC, et al. Acute large bowel obstruction. Comparison of county, Veterans Administration, and community hospital populations. Arch Surg. 1974;108:470-476.

24. Hadjis NS, McAuley G, Ruo L, et al. Acute abdominal pain and the urge to defecate in the young and old: a useful symptom complex? J Emerg Med. 1999;17:239-242.

25. Ramoska EA, Sacchetti AD, Nepp M. Reliability of patient history in determining the possibility of pregnancy. Ann Emerg Med. 1989;18: $48-50$.

26. McNamara R. Abdominal pain in the elderly. In: Tintinalli JE, Kelen GD, Stapczynski S, et al, editors. Emergency Medicine: a Comprehensive Study Guide. 6th ed. New York: McGraw-Hill; 2004:515-519.

27. Trowbridge RL, Ruttconski NK, Shojania KG. Does this patient have acute cholecystitis? JAMA. 2003;289:80-86.

28. Silen W. Method of diagnosis: the examination of the patient. In: Cope's Early Diagnosis of the Acute Abdomen. New York: Oxford; 2010:28-40.

29. Bundy DG, Byerley JS, Liles EA, et al. Does this child have appendicitis? JAMA. 2007;298:438-451.

30. Meyerowitz BR. Abdominal palpation by stethoscope [letter]. Arch Surg. 1976;111:831.

31. Marston WA, Ahlquist R, Johnson G, et al. Misdiagnosis of ruptured abdominal aortic aneurysms. J Vasc Surg. 1992;16:17-22.

32. Klompas M. Does this patient have an acute thoracic aortic dissection? JAMA. 2002;287:2262-2272.

33. Prout WG. The significance of rebound tenderness in the acute abdomen. Br J Surg. 1970;57:508-510.

34. Liddington MI, Thomson WHF. Rebound tenderness test. Br J Surg. 1991;78:795-796.

35. Bennett DH, Tambeur LJ, Campbell WB. Use of coughing test to diagnose peritonitis. BMJ. 1994;308:1336.

36. Markle GB. Heel-drop jarring test for appendicitis. Arch Surg. 1985;120:243.

37. Brewster GS, Herbert ME. Medical myth: a digital rectal examination should be performed on all individuals with suspected appendicitis. West J Med. 2000;173:207-208.

38. Jesudason EC, Walker J. Rectal examination in paediatric surgical practice. Br J Surg. 1999;86:376-378. 
39. Thomson H, Francis DMA. Abdominal-wall tenderness: a useful sign in the acute abdomen. Lancet. 1977;2:1053-1054.

40. Gray DW, Seabrook G, Dixon JM, et al. Is abdominal wall tenderness a useful sign in the diagnosis of non-specific abdominal pain? Ann $R$ Coll Surg Engl. 1988;70:233-234.

41. Thomson WH, Dawes RF, Carter SS. Abdominal wall tenderness: a useful sign in chronic abdominal pain. Br J Surg. 1991;78:223-225.

42. Kovachev LS. 'Cough sign': a reliable test in the diagnosis of intraabdominal inflammation [letter]. Br J Surg. 1994;81:1541.

43. Jeddy TA, Vowles RH, Southam JA. 'Cough sign': a reliable test in the diagnosis of intra-abdominal inflammation. Br J Surg. 1994;81:279.

44. Gray DW, Dixon JM, Collin J. The closed eyes sign: an aid to diagnosing nonspecific abdominal pain. BMJ. 1988;297:837.

45. Aldea PA, Meehan JP, Sternbach G. The acute abdomen and Murphy's signs. J Emerg Med. 1986;4:57-63.

46. Kharbanda AB, Taylor GA, Fishman SJ, et al. A clinical decision rule to identify children at low risk for appendicitis. Pediatrics. 2005;116: 709-716.

47. Brownfield E. Pain management. Use of analgesics in the acute abdomen. In: Making Health Care Safer: a critical analysis of patient safety practices. Evidence Report/Technology Assessment, No 43. AHRQ Publication No 01-E058. Rockville (MD): Agency for Healthcare Research and Quality; 2001:396-400. Available from: http:// www.ahrq.gov/clinic/ptsafety/. Accessed February 26, 2011.
48. Silen W. Principles of diagnosis in acute abdominal disease. In: Cope's Early Diagnosis of the Acute Abdomen. New York: Oxford; 2010:3-17.

49. Thomas SH, Silen WH, Cheema F, et al. Effects of morphine analgesia on diagnostic accuracy in emergency department patients with abdominal pain: a prospective, randomized trial. J Am Coll Surg. 2003;196: $18-31$.

50. Valentine RJ, Barth M, Myers S, et al. Nonvascular emergencies presenting as ruptured abdominal aortic aneurysms. Surgery. 1993; 113:286-289.

51. Kessler N, Cyteval C, Gallix B, et al. Appendicitis: evaluation of sensitivity, specificity, and predictive value of US, Doppler US, and laboratory findings. Radiology. 2004;230:472-478.

52. Gwynn LK. The diagnosis of acute appendicitis: clinical assessment versus computed tomography evaluation. J Emerg Med. 2001;21: $119-123$.

53. Lee SL, Walsh AJ, Ho HS. Computed tomography and ultrasonography do not improve and may delay the diagnosis and treatment of acute appendicitis. Arch Surg. 2001;136:556-562.

54. Smith JE, Hall EJ. The use of plain abdominal $x$ rays in the emergency department. Emerg Med J. 2009;26:160-163.

55. Maull KI, Reath DB. Pneumogastrography in the diagnosis of perforated peptic ulcer. Am J Surg. 1984;148:340-345.
International Journal of General Medicine

\section{Publish your work in this journal}

The International Journal of General Medicine is an international, peer-reviewed open-access journal that focuses on general and internal medicine, pathogenesis, epidemiology, diagnosis, monitoring and treatment protocols. The journal is characterized by the rapid reporting of reviews, original research and clinical studies across all disease areas.

\section{Dovepress}

A key focus is the elucidation of disease processes and management protocols resulting in improved outcomes for the patient.The manuscript management system is completely online and includes a very quick and fair peer-review system. Visit http://www.dovepress.com/ testimonials.php to read real quotes from published authors. 